\title{
Influence of fertilization and soil tillage on nitrogen uptake and utilization efficiency of quinoa crop (Chenopodium quinoa Willd.)
}

\author{
Ioanna P. Kakabouki ${ }^{1 *}$, Dimitra Hela ${ }^{2}$, Ioannis Roussis ${ }^{1}$, Panagiota Papastylianou ${ }^{1}$, Adriana \\ F. Sestras ${ }^{3}$. Dimitrios J. Bilalis ${ }^{1}$
}

\begin{abstract}
${ }^{1}$ Agricultural University of Athens, School of Agriculture, Engineering and Environmental Sciences, Department of Crop Science, Laboratory of Agronomy, 75 Iera Odos str., 11855 Athens, Greece. ${ }^{2}$ University of Ioannina, School of Sciences, Department of Chemistry, University of Ioannina, 45110 Ioannina, Greece. ${ }^{3}$ University of Agricultural Sciences and Veterinary Medicine of Cluj-Napoca, Faculty of Horticulture, 3-5 Manastur St., 400372, Cluj-Napoca, Romania. *Corresponding author: i.kakabouki@gmail.com
\end{abstract}

\begin{abstract}
In recent years, quinoa (Chenopodium quinoa Willd.) has attached the interest as a multi-purpose crop. A 3-year field experiment was conducted to determine the effects of tillage systems and fertilization on nitrogen uptake and use efficiency of quinoa crop. The experiment was laid out in a split-plot design with two replicates, two main plots [conventional and minimum tillage] and four sub-plots (fertilization treatments: untreated (control), inorganic fertilization of 100 (N1) and $200 \mathrm{~kg} \mathrm{~N} \mathrm{ha}^{-1}$ (N2) and sheep manure). The results indicated that nitrogen uptake and utilization efficiency were only influenced by fertilization. The higher biomass nitrogen content (4.08-4.33\%), biomass nitrogen yield (371-386 $\mathrm{kg} \mathrm{N} \mathrm{ha}^{-1}$ ), seed nitrogen content (2.59-2.78\%), seed nitrogen yield (62.58-65.42 $\left.\mathrm{kg} \mathrm{N} \mathrm{ha}^{-1}\right)$ and total plant nitrogen uptake (437.20-454.93 $\mathrm{kg} \mathrm{N}^{-1}$ ) were found in $\mathrm{N} 2$. Nitrogen harvest index and nitrogen utilization efficiency were up to $60 \%$ lower and $40 \%$ lower, respectively, in inorganic treatments than in the control. Rates of nitrogen higher than $100 \mathrm{~kg} \mathrm{~N} \mathrm{ha}^{-1}$ (N1) did not increase the nitrogen agronomic efficiency. Regarding apparent nitrogen recovery efficiency, the higher values observed under inorganic fertilization and being greater than $100 \%$. The highest rates of change of nitrate reduction in soil $\left(-0.108\right.$ to $-0.188 \mathrm{~N} \%$ day $\left.^{-1}\right)$ and nitrogen increase in plant $\left(0.025\right.$ to $0.027 \mathrm{~N} \%$ day $\left.^{-1}\right)$ were observed under $\mathrm{N} 2$ treatment. As a conclusion, quinoa has a high capacity to take up nitrate from the soil, but presents lower nitrogen remobilization from the vegetative parts into the seeds under high nitrogen supply.
\end{abstract}

Keywords: Fertilization, nitrogen response, NUE indices, quinoa, tillage 


\section{Introduction}

Quinoa (Chenopodium quinoa Willd.) is a pseudocereal crop that has constituted an important part of the food of the Andean population for thousands of years due to its high nutritional value (Vega-Gálvez et al. 2010) and its adaptability to grow with less inputs and water and to tolerate a variety of biotic and abiotic stresses (Jacobsen, 2003). The grain has a high protein content with balanced presence of essential amino acids such as lysine and a wide range of vitamins, minerals and saponins (Koziol, 1992; Bilalis et al., 2012). Quinoa is often used similarly to cereals, but unlike these crops, quinoa seeds have a better balance in the amino acid composition and are gluten free (Thanapornpoonpong et al., 2008). Moreover, the whole plant can be used as green fodder for animals (Kakabouki et al., 2014). As a multi-purpose crop with potential uses for both human and animal consumption and nutrition, quinoa has also attracted the interest as a food security crop outside the center of origin in several countries around the globe (especially in Europe), initiating introduction and research work on it (Jacobsen, 2003; Benlhabib et al. 2014). The Food and Agriculture Organization of the United Nations (FAO) has declared the year 2013 as the year of quinoa.

Nitrogen is a key limiting element for plant growth, which is also the most complex one considering all of the potential forms and processes in its cycle (Montemurro and Diacono, 2016). It is strongly involved in all of the plant metabolic processes and its rate of uptake and partition is largely determined by supply and demand during the various stages of plant growth (Delogu et al., 1998). Availability and supply of nitrogen vary between crop species and depend on their requirements (Sinclair and de Wit, 1975). The positive effect of nitrogen on quinoa has been proven by several studies on growth and grain yield. Jacobsen et al. (1994) reported that yield response of quinoa at $40 \mathrm{~kg}$
$\mathrm{N} \mathrm{ha}^{-1}$ was $24.1 \%$ lower than at $160 \mathrm{~kg} \mathrm{~N} \mathrm{ha}^{-1}$. According to Schulte auf'm Erley et al. (2005), the grain yield of quinoa (cv. Faro and Cochabamba) almost doubled from $1790 \mathrm{~kg} \mathrm{ha}^{-1}$ under the unfertilized treatment to $3495 \mathrm{~kg} \mathrm{ha}^{-1}$ under $120 \mathrm{~kg} \mathrm{~N} \mathrm{ha}^{-1}$. Additionally, in a greenhouse study, quinoa has shown a positive reaction to nitrogen and specifically, biomass, harvest index and protein content were positively correlated with nitrogen fertilization (Thanapornpoonpong, 2004).

Besides increasing grain yield, nitrogen is also responsible for an important part of agriculture-related pollution through nitrate $\left(\mathrm{NO}_{3}^{-}\right)$leaching, ammonia $\left(\mathrm{NH}_{3}\right)$ volatilization and nitrous oxide $\left(\mathrm{N}_{2} \mathrm{O}\right)$ emissions and for this reason the efficiency of fertilizer use has to be considered (Fagaria and Baligar, 2005; Schulte auf'm Erley et al., 2005; Lobos Ortega et al., 2016). Tremendous losses of 40 to $60 \%$ of the applied nitrogen occur due to uncontrolled and unreasonable use of nitrogen fertilizers, which may affect crop yields (Craswell and Godwin, 1984). Nitrogen use efficiency (NUE) can been defined as the maximum economic yield produced per unit of nitrogen applied, absorbed or utilized by the plant to produce grain and biomass, and is an important approach in evaluating the absorption of applied fertilizers by plants and their role in crop yields (Fageria and Baligar, 2005). In the literature, NUE has been defined and calculated in several ways. Fageria and Baligar (2005) described five definitions of calculating NUE in crops, which are grouped or classified as agronomic efficiency $(\mathrm{AE})$, physiological efficiency (PE), agro-physiological efficiency (APE), apparent recovery efficiency (ARE) and utilization efficiency (UE). The increase in NUE and grain yield mainly depend on timely planting, proper tillage and optimum nitrogen rate and management (Zemenchik and Albrecht, 2002). The main goal in improving nitrogen utilization, optimizing the fertilization and reducing the risk of surface and belowground water 
resources contamination is to better understand the plant nitrogen response (Mahler et al., 1994).

Nitrogen harvest index (NHI) is defined as the partitioning of total plant nitrogen into grain. It consists an important index, which provides an indication of how efficiently the plant utilized acquired nitrogen for grain production and varied from crop species to crop species and among different genotypes of the same species (Fageria and Baligar, 2005).

There was no information about the effects of tillage systems and fertilization on nitrogen efficiency in quinoa crop production under Mediterranean semi-arid conditions. Therefore, the aim of the present study was to investigate the effect of tillage system and fertilization on nitrogen uptake and its assimilation from the soil to vegetative parts and grains of quinoa.

\section{Materials and Methods}

A quinoa crop (Chenopodium quinoa Willd. cv. Faro) was established in Western Greece (Agrinio region, Latitude: $38^{\circ} 35^{\prime} \mathrm{N}$, Longitude: $21^{\circ} 25^{\prime} \mathrm{E}$ ) from 2011 to 2013. The soil was a clay loam $(24.9 \%$ clay, $61.2 \%$ silt, $13.9 \%$ sand) with $\mathrm{pH}\left(1: 2 \mathrm{H}_{2} \mathrm{O}\right) 7.4$, EC $0.63 \mathrm{mS}$ $\mathrm{cm}^{-1}, 0.152 \%$ total nitrogen, a sufficient supply of phosphorus (P Olsen: $175 \mathrm{mg} \mathrm{kg}^{-1}$ soil) and potassium (632 $\mathrm{mg} \mathrm{kg}^{-1}$ soil) and $1.45 \%$ organic matter content. The site was managed according to organic agricultural guidelines (EC 834/2007).

Meteorological data concerning air temperature and precipitation of the experimental site during the experimental periods are presented in Figure 1.

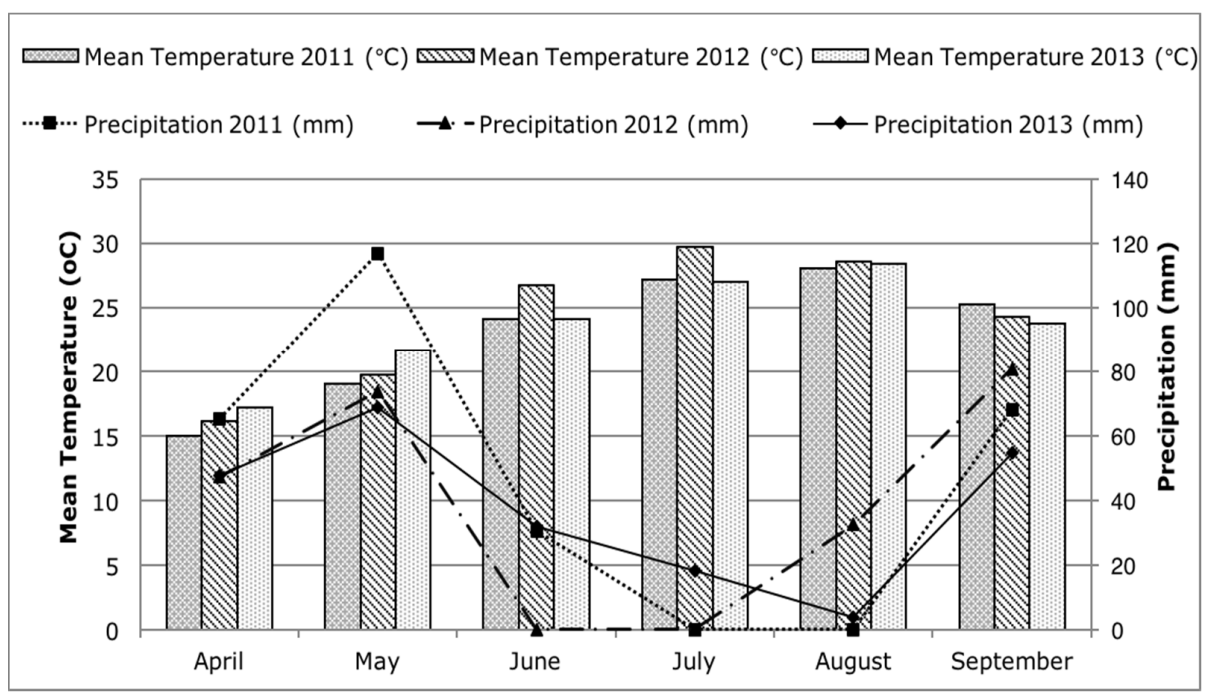

Figure 1. Meteorological data (mean monthly air temperature and precipitation) for experimental site during the experimental periods (April-September, 2011, 2012 and 2013). 
The experiment was set up on an area of $850 \mathrm{~m}^{2}$ according to the split plot design with two replicates, two main plots (conventional tillage: CT, moldboard plowing at $25 \mathrm{~cm}$, followed by one rotary hoeing at 10-15 $\mathrm{cm}$; minimum tillage: MT, chiseling at $25 \mathrm{~cm}$ depth followed by chiseling at $10-15 \mathrm{~cm}$ ) and four sub-plots [fertilization treatments: control, sheep manure (3000 $\mathrm{kg} \mathrm{ha}^{-1}$, solid, $11.52 \% \mathrm{~N}$ ), inorganic fertilization (fertilizer 26-0-0) with $100 \mathrm{~kg} \mathrm{~N} \mathrm{ha}^{-1}(\mathrm{~N} 1)$ and $200 \mathrm{~kg} \mathrm{~N}$ $\mathrm{ha}^{-1}$ (N2)]. The main plot size was $200 \mathrm{~m}^{2}$. Quinoa was sown by hand in rows $30 \mathrm{~cm}$ apart at a depth of $2-3 \mathrm{~cm}$ and approximate density of 250,000 plants $\mathrm{ha}^{-1}$. The field was sown on 6th April 2011, $5^{\text {th }}$ April 2012 and $10^{\text {th }}$ April 2013. Overhead sprinkler system was also set up on the field. The total quantity of water applied during the cultivation periods was 230,250 and 250 $\mathrm{mm}$ in 2011, 2012 and 2013, respectively.

Five plant samples were randomly selected from each plot at 25, 50, 75, 100, 125 and 150 Days After Sowing (DAS). The plants collected were divided into stems, inflorescences, grains, green and yellow leaves, weighted and oven-dried for $48 \mathrm{~h}$ at $65^{\circ} \mathrm{C}$. All plant samples were ground to fine powder and used for determination of total nitrogen. The total nitrogen was determined using Kjeldahl procedure. Total plant nitrogen uptake was calculated as nitrogen in the total above-ground (aerial biomass + grain) dry matter at time of maturity (150 DAS).

Nitrogen harvest index (NHI) was calculated as given by Ye et al. (2007) as defined in Formula 1:

$$
N H I=\frac{\text { grain } N \text { uptake }(\mathrm{kg})}{\text { Total plan } N \text { uptake }(\mathrm{kg})} \quad \text { Formula } 1
$$

Nitrogen use efficiency (NUE) was expressed by the indices, apparent nitrogen recovery efficiency (ANRE), nitrogen utilization efficiency (NUtE) and nitrogen agronomic efficiency (NAE) that were determined as described by Fageria and Baligar (2005) and Ye et al. (2007) and defined as:

$$
\operatorname{ANRE}(\%)=\frac{[(\text { total } N \text { uptake of the fertilized plot }(\mathrm{kg})-\text { total } N \text { uptake of the unfertilized plot }(\mathrm{kg}))}{\text { quantity of } N \text { applied }(\mathrm{kg})]} \times 100
$$

$$
N U t E=\frac{\text { grain yield }(\mathrm{kg})}{\text { total plant } N \text { uptake }(\mathrm{kg})}
$$

$$
N A E=\frac{(\text { grain yield of the fertilized plot }(\mathrm{kg})-\text { grain yield of the unfertilized plot }(\mathrm{kg}))}{\text { quantity of } N \text { applied }(\mathrm{kg})}
$$


For the determination of soil nitrate concentration, soil samples were taken by a hand operated soil auger at depth interval of 0-15 cm. Three samples were collected from each plot at 25, 50, 75, 100, 125 and 150 DAS. The soil was air-dried, milled and passed through $2 \mathrm{~mm}$ sieves. Extraction of nitrate from soil samples was carried out using a $2 \mathrm{~N} \mathrm{KCl}$ solution. Nitrate content was measured by the cadmium reduction method at an absorbance of $540 \mathrm{~nm}$ (Keeney and Nelson, 1982).

Finally, the rate of change of nitrogen content in plant $\left(\mathrm{N} \%\right.$ day $\left.^{-1}\right)$ and rate of change of nitrates in soil (ppm $\mathrm{NO}_{3}-$ day $^{-1}$ ) were also evaluated during the experimental periods.

The data were checked for normality and subjected to statistical analysis according to the split-plot design. The statistical analysis was performed with SigmaPlot 12 statistical software (Systat Software Inc., San Jose, CA, USA). The Tukey's test was used to detect and separate the mean treatment differences. All comparisons were made at the $5 \%$ level of significance.

\section{Results}

The effects of the tillage system and fertilization on the biomass nitrogen content of quinoa are presented in Table 1. Analysis of variance (Table 6) generally revealed that biomass nitrogen content was not affected by the tillage system, although there was a tendency for biomass nitrogen content to be lower under conventional tillage than under minimum tillage. The only significant difference between the different tillage systems was observed in the third year of the experiment, especially at 50 and $100 \mathrm{DAS}$, where plants of minimum tillage plots had statistically higher values (1.31 and $3.34 \%$, respectively), while the lowest values (1.11 and $3.10 \%$, respectively) were obtained in conventional plots. During the experiment, the increase in nitrogen content was continued until 125 days after sowing (DAS) and showed stabilization at 150 DAS. Biomass nitrogen content was significantly affected by different fertilization treatments. All fertilization treatments resulted in higher values than those of the control. The highest nitrogen content was observed at 150 DAS for all years. The greatest values (4.02-4.33\%) was found in N2 treatment.

Concerning the nitrogen yield of biomass, there were no significant differences between conventional and minimum tillage systems, although, with the exception of the first year (2011), nitrogen yield was slightly higher in conventional tillage plots. As shown in Table 2 and according to the combined analysis of variance (Table 6) the nitrogen yield in above-ground parts of plant was only affected by fertilization. In particular,

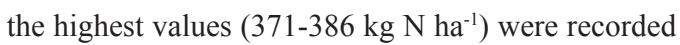
in N2 treatment, while the lowest were obtained in untreated plots (193-217 $\left.\mathrm{kg} \mathrm{N} \mathrm{ha}^{-1}\right)$.

Seed nitrogen content was only affected by fertilization. Specifically, the highest seed nitrogen content (2.59-2.78\%) was found in the case of N2 treatment. Seed nitrogen content had positive and significant correlation with soil nitrate content and biomass nitrogen content ( $r=0.544, p<0.001$ and $r=0.720, p<0.001$, respectively).

The results of the experiment indicated that seed nitrogen yield was not affected by the tillage system during the experimental periods. Despite the absence of significant effects of tillage systems on seed nitrogen yield, the plots subjected under conventional tillage presented higher values (53.73-58.72 $\left.\mathrm{kg} \mathrm{N} \mathrm{ha}^{-1}\right)$, while the lowest values (52.58-57.79 $\mathrm{kg} \mathrm{ha}^{-1}$ ) were obtained from plots under minimum tillage. With the exception of the first year, seed nitrogen yield was actually influenced by different fertilization treatments. The highest yield was achieved in N2 treatment with the values being 62.58 (29\% higher than control) and $65.42 \mathrm{~kg} \mathrm{~N} \mathrm{ha}^{-1}$ (61\% higher than control) in 2012 and 2013, respectively. 
Table 1. Effects of tillage systems (conventional and minimum tillage: CT and MT, respectively) and fertilization (control, inorganic fertilization $100 \mathrm{~kg} \mathrm{~N} \mathrm{ha}^{-1}(\mathrm{~N} 1)$, inorganic fertilization $200 \mathrm{~kg} \mathrm{~N}^{-1}(\mathrm{~N} 2)$ and sheep manure) on biomass nitrogen content ( $\mathrm{N} \%)$.

Tillage System

\begin{tabular}{|c|c|c|c|c|c|c|c|c|c|c|c|c|}
\hline \multirow[b]{2}{*}{ Fertilization } & \\
\hline & CT & MT & CT & MT & CT & MT & CT & MT & CT & MT & CT & MT \\
\hline \multirow[t]{2}{*}{2011} & \multicolumn{12}{|c|}{ Biomass Nitrogen Content (N\%) } \\
\hline & \multicolumn{2}{|c|}{25 DAS } & \multicolumn{2}{|c|}{$50 \mathrm{DAS}$} & \multicolumn{2}{|c|}{75 DAS } & \multicolumn{2}{|c|}{100 DAS } & \multicolumn{2}{|c|}{125 DAS } & \multicolumn{2}{|c|}{150 DAS } \\
\hline Control & 0.75 & 0.73 & 1.05 & 1.11 & 1.71 & 2.08 & 2.03 & 2.53 & 2.38 & 2.71 & 2.39 & 2.71 \\
\hline N1 & 0.94 & 0.97 & 1.50 & 1.67 & 2.24 & 2.61 & 2.89 & 3.22 & 3.17 & 3.56 & 3.22 & 3.52 \\
\hline $\mathrm{N} 2$ & 1.04 & 1.06 & 2.05 & 2.47 & 3.05 & 3.24 & 3.61 & 3.98 & 3.90 & 4.33 & 4.08 & 4.36 \\
\hline Manure & 0.75 & 0.76 & 1.23 & 1.17 & 1.96 & 2.19 & 2.44 & 2.52 & 2.66 & 2.77 & 2.60 & 2.81 \\
\hline $\mathrm{F}_{\text {tillage }}$ & \multicolumn{2}{|c|}{$0.19 \mathrm{~ns}$} & \multicolumn{2}{|c|}{$3.02 \mathrm{~ns}$} & \multicolumn{2}{|c|}{$4.55 \mathrm{~ns}$} & \multicolumn{2}{|c|}{$3.72 \mathrm{~ns}$} & \multicolumn{2}{|c|}{$3.61 \mathrm{~ns}$} & \multicolumn{2}{|c|}{$2.88 \mathrm{~ns}$} \\
\hline $\mathrm{F}_{\text {fertilization }}$ & \multicolumn{2}{|c|}{$\begin{array}{l}28.14 * * * \\
\text { (Tukey's test } \\
=0.102 \text { ) }\end{array}$} & \multicolumn{2}{|c|}{$\begin{array}{l}39.82 * * * \\
\text { (Tukey's test } \\
=0.400)\end{array}$} & \multicolumn{2}{|c|}{$\begin{array}{l}16.35^{* * *} \\
\text { (Tukey's test } \\
=0.595 \text { ) }\end{array}$} & \multicolumn{2}{|c|}{$\begin{array}{l}16.92 * * * \\
\text { (Tukey's test } \\
=0.711)\end{array}$} & \multicolumn{2}{|c|}{$\begin{array}{c}17.95 * * * \\
(\text { Tukey's test }= \\
0.712)\end{array}$} & \multicolumn{2}{|c|}{$\begin{array}{c}21.40 * * * \\
(\text { Tukey's test }= \\
0.659)\end{array}$} \\
\hline $\begin{array}{l}F_{\text {tillage } X} \\
\text { fertilization }\end{array}$ & \multicolumn{2}{|c|}{$0.16 \mathrm{~ns}$} & \multicolumn{2}{|c|}{$1.46 \mathrm{~ns}$} & \multicolumn{2}{|c|}{$0.12 \mathrm{~ns}$} & \multicolumn{2}{|c|}{$0.29 \mathrm{~ns}$} & \multicolumn{2}{|c|}{$0.18 \mathrm{~ns}$} & & \\
\hline 2012 & & & & & & Iass $\mathrm{Ni}$ & gen $\mathrm{C}$ & tent $(\mathrm{N}$ & & & & \\
\hline & 25 & & 50 & & & & 100 & AS & & AS & 150 & \\
\hline Control & 0.70 & 0.77 & 1.14 & 1.13 & 1.72 & 1.94 & 2.16 & 2.30 & 2.45 & 2.42 & 2.47 & 2.48 \\
\hline N1 & 0.90 & 0.93 & 1.67 & 1.80 & 2.29 & 2.64 & 2.97 & 3.10 & 3.64 & 3.39 & 3.70 & 3.44 \\
\hline $\mathrm{N} 2$ & 0.99 & 0.98 & 2.05 & 2.09 & 2.67 & 2.83 & 3.57 & 3.70 & 4.25 & 4,32 & 4.33 & 4.33 \\
\hline Manure & 0.75 & 0.77 & 1.38 & 1.24 & 2.05 & 2.32 & 2.57 & 2.78 & 3.21 & 3.38 & 3.37 & 3.47 \\
\hline $\mathrm{F}_{\text {tillage }}$ & & & 0.0 & & & & & & & & & \\
\hline $\mathrm{F}_{\text {fertilization }}$ & $\begin{array}{r}14 . \\
\text { (Tuke } \\
=0\end{array}$ & $\begin{array}{l}* * \\
\text { s test } \\
18)\end{array}$ & $\begin{array}{r}33 . \\
\text { (Tuke } \\
=0\end{array}$ & $\begin{array}{l}* * * \\
\text { s test } \\
77)\end{array}$ & $\begin{array}{r}4 \\
\text { (Tuk } \\
=(\end{array}$ & $\begin{array}{l}* \\
\text { s test } \\
12)\end{array}$ & $\begin{array}{r}24 . \\
\text { (Tuk } \\
=0\end{array}$ & $\begin{array}{l}* * * \\
\text { s test } \\
56)\end{array}$ & $\begin{array}{r}10 \\
\text { (Tuk } \\
\text { ( }\end{array}$ & $\begin{array}{l}* * * \\
\text { test }= \\
8)\end{array}$ & $\begin{array}{r}104 \\
\text { (Tuke } \\
0 .\end{array}$ & $\begin{array}{l}* * * \\
\text { test }= \\
3)\end{array}$ \\
\hline $\begin{array}{l}F_{\text {tillage } X} \\
\text { fertilization }\end{array}$ & & & & & & & & & & & & \\
\hline 2013 & & & & & & $\operatorname{ass~} \mathrm{Ni}$ & gen $\mathrm{C}$ & tent $(\mathrm{N}$ & & & & \\
\hline & 25 & & 50 & & & AS & 100 & AS & & AS & 150 & AS \\
\hline Control & 0.53 & 0.48 & 0.87 & 0.99 & 1.60 & 1.65 & 1.94 & 2.28 & 2.34 & 2.41 & 2.40 & 2.46 \\
\hline N1 & 0.78 & 0.87 & 1.25 & 1.42 & 1.99 & 2.24 & 2.82 & 2.99 & 3.24 & 3.71 & 3.29 & 3.68 \\
\hline $\mathrm{N} 2$ & 0.90 & 0.90 & 1.34 & 1.67 & 2.30 & 2.49 & 3.10 & 3.24 & 3.96 & 4.12 & 4.02 & 4.15 \\
\hline Manure & 0.63 & 0.71 & 0.98 & 1.18 & 1.77 & 1.93 & 2.19 & 2.62 & 2.89 & 3.12 & 2.96 & 3.17 \\
\hline $\mathrm{F}_{\text {tillage }}$ & & & $\begin{array}{r}8 . \\
\text { (Tuke } \\
=0\end{array}$ & $\begin{array}{l}3^{*} \\
\text { 's test } \\
82)\end{array}$ & & & $\begin{array}{r}12 \\
\text { (Tuk } \\
=0\end{array}$ & $\begin{array}{l}* * * \\
\text { s test } \\
97)\end{array}$ & & & & \\
\hline $\mathrm{F}_{\text {fertilization }}$ & $\begin{array}{r}12 . \\
\text { (Tuke } \\
=0\end{array}$ & $\begin{array}{l}* * \\
\text { s test } \\
84)\end{array}$ & $\begin{array}{r}13 . \\
\text { (Tuke } \\
=0\end{array}$ & $\begin{array}{l}* * \\
\text { s test } \\
60)\end{array}$ & $\begin{array}{r}4 \\
\text { (Tuk } \\
=(\end{array}$ & $\begin{array}{l}* \\
\text { s test } \\
51)\end{array}$ & $\begin{array}{r}39 . \\
\text { (Tuk } \\
=0\end{array}$ & $\begin{array}{l}* * * \\
\text { s test } \\
25)\end{array}$ & $\begin{array}{r}19 \\
\text { (Tuk } \\
\text { ( }\end{array}$ & $\begin{array}{l}* * * * \\
\text { test }= \\
8)\end{array}$ & $\begin{array}{r}21 . \\
\text { (Tuke } \\
0 .\end{array}$ & $\begin{array}{l}* * * \\
\text { test }= \\
1)\end{array}$ \\
\hline $\begin{array}{l}F_{\text {tillage } X} \\
\text { fertilization }\end{array}$ & & & & & & & & & & & & \\
\hline
\end{tabular}

F-test ratios are from ANOVA. Significant at $* * *$ and $* * *$ indicate significance at $\mathrm{p}=0.05,0.01$ and 0.001 , respectively and ns: not significant. The Tukey's test $(\mathrm{p}=0.05)$ values for tillage system and fertilization are also presented. 
Table 2. Effects of tillage systems (conventional and minimum tillage: $\mathrm{CT}$ and MT, respectively) and fertilization (control, inorganic fertilization $100 \mathrm{~kg} \mathrm{~N} \mathrm{ha}^{-1}(\mathrm{~N} 1)$, inorganic fertilization $200 \mathrm{~kg} \mathrm{~N}^{-1}$ (N2) and sheep manure) on biomass nitrogen yield $\left(\mathrm{kg} \mathrm{N} \mathrm{ha}^{-1}\right)$, total plant nitrogen uptake $\left(\mathrm{kg} \mathrm{Nha}^{-1}\right)$, seed nitrogen content $(\% \mathrm{~N})$ and seed nitrogen yield $\left(\mathrm{kg} \mathrm{Nha}^{-1}\right)$.

\begin{tabular}{|c|c|c|c|c|c|c|c|c|}
\hline \multirow[b]{2}{*}{ Fertilization } & \multicolumn{8}{|c|}{ Tillage System } \\
\hline & $\mathrm{CT}$ & MT & $\mathrm{CT}$ & MT & $\mathrm{CT}$ & MT & $\mathrm{CT}$ & MT \\
\hline 2011 & \multicolumn{2}{|c|}{$\begin{array}{l}\text { Biomass Nitrogen Yield } \\
\qquad\left(\mathrm{kg} \mathrm{N} \mathrm{ha}^{-1}\right)\end{array}$} & \multicolumn{2}{|c|}{$\begin{array}{c}\text { Total Plant Nitrogen } \\
\text { Uptake } \\
\left(\mathrm{kg} \mathrm{N} \mathrm{ha}^{-1}\right)\end{array}$} & \multicolumn{2}{|c|}{$\begin{array}{c}\text { Seed Nitrogen Content } \\
(\% \mathrm{~N})\end{array}$} & \multicolumn{2}{|c|}{$\begin{array}{l}\text { Seed Nitrogen Yield } \\
\left(\mathrm{kg} \mathrm{N} \mathrm{ha}^{-1}\right)\end{array}$} \\
\hline Control & 208.36 & 258.43 & 258.43 & 226.22 & 2.07 & 2.11 & 50.07 & 46.82 \\
\hline N1 & 285.51 & 342.41 & 342.41 & 296.35 & 2.26 & 2.26 & 56.90 & 52.27 \\
\hline $\mathrm{N} 2$ & 374.97 & 440.74 & 440.74 & 381.61 & 2.60 & 2.58 & 65.77 & 59.38 \\
\hline Manure & 237.62 & 298.97 & 298.97 & 239.97 & 2.30 & 2.17 & 61.36 & 51.86 \\
\hline $\mathrm{F}_{\text {tillage }}$ & \multicolumn{2}{|c|}{$0.20 \mathrm{~ns}$} & \multicolumn{2}{|c|}{$0.02 \mathrm{~ns}$} & \multicolumn{2}{|c|}{$0.20 \mathrm{~ns}$} & \multicolumn{2}{|c|}{$2.78 \mathrm{~ns}$} \\
\hline $\mathrm{F}_{\text {fertilization }}$ & \multicolumn{2}{|c|}{$11.59^{* *}$} & Tukey's test $=81.475$ ) & $10.60 * *$ & \multicolumn{2}{|c|}{$\begin{array}{c}11.27 * * \\
(\text { Tukey's test }=0.230)\end{array}$} & \multicolumn{2}{|c|}{$2.68 \mathrm{~ns}$} \\
\hline$F_{\text {tillage } X \text { fertilization }}$ & \multicolumn{2}{|c|}{$0.03 \mathrm{~ns}$} & \multicolumn{2}{|c|}{$0.04 \mathrm{~ns}$} & \multicolumn{2}{|c|}{$0.33 \mathrm{~ns}$} & \multicolumn{2}{|c|}{$0.14 \mathrm{~ns}$} \\
\hline 2012 & Biomass & n Yield & $\begin{array}{r}\text { Total F } \\
(\mathrm{k} \\
\end{array}$ & $\begin{array}{l}\text { Nitrogen } \\
\text { ake } \\
\left.\mathrm{ha}^{-1}\right)\end{array}$ & Seed Ni & Content & \multicolumn{2}{|c|}{$\begin{array}{l}\text { Seed Nitrogen Yield } \\
\left(\mathrm{kg} \mathrm{N} \mathrm{ha}^{-1}\right)\end{array}$} \\
\hline Control & 202.32 & 249.70 & 249.70 & 198.71 & 2.01 & 2.14 & 47.39 & 44.64 \\
\hline N1 & 321.06 & 377.97 & 377.97 & 288.52 & 2.30 & 2.50 & 56.91 & 57.51 \\
\hline N2 & 396.95 & 466.90 & 466.90 & 377.03 & 2.70 & 2.87 & 69.95 & 65.92 \\
\hline Manure & 308.62 & 368.27 & 368.27 & 312.66 & 2.26 & 2.30 & 59.66 & 57.53 \\
\hline $\mathrm{F}_{\text {tillage }}$ & \multicolumn{2}{|c|}{$2.11 \mathrm{~ns}$} & \multicolumn{2}{|c|}{$1.97 \mathrm{~ns}$} & \multicolumn{2}{|c|}{$3.98 \mathrm{~ns}$} & \multicolumn{2}{|c|}{$0.37 \mathrm{~ns}$} \\
\hline$F_{\text {fertilization }}$ & $\begin{array}{r}7 \\
\text { (Tukey' }\end{array}$ & $38.560)$ & \multicolumn{2}{|c|}{$\begin{array}{c}65.53 * * * \\
(\text { Tukey's test }= \\
44.220)\end{array}$} & \multicolumn{2}{|c|}{$\begin{array}{c}19.08 * * * \\
(\text { Tukey's test }=0.294)\end{array}$} & \multicolumn{2}{|c|}{$\begin{array}{c}6.86^{*} \\
(\text { Tukey’s test }= \\
12.200)\end{array}$} \\
\hline$F_{\text {tillage } X \text { fertilization }}$ & \multicolumn{2}{|c|}{$0.84 \mathrm{~ns}$} & \multicolumn{2}{|c|}{$0.53 \mathrm{~ns}$} & & & & \\
\hline 2013 & Biomass & n Yield & $\begin{array}{r}\text { Total F } \\
(\mathrm{k} \\
\end{array}$ & $\begin{array}{l}\text { Nitrogen } \\
\text { ake } \\
\left.\text { ha }^{-1}\right)\end{array}$ & Seed Ni & Content & $\begin{array}{r}\text { Seed N } \\
(\mathrm{k}\end{array}$ & $\begin{array}{l}\text { en Yield } \\
a^{-1} \text { ) }\end{array}$ \\
\hline Control & 194.30 & 194.30 & 194.30 & 194.30 & 2.11 & 2.09 & 41.41 & 39.66 \\
\hline N1 & 285.27 & 285.27 & 285.27 & 285.27 & 2.50 & 2.42 & 54.61 & 54.52 \\
\hline $\mathrm{N} 2$ & 366.58 & 366.58 & 366.58 & 366.58 & 2.76 & 2.74 & 65.58 & 65.26 \\
\hline Manure & 269.36 & 269.36 & 269.36 & 269.36 & 2.58 & 2.40 & 53.34 & 52.43 \\
\hline $\mathrm{F}_{\text {tillage }}$ & & & & & & & & \\
\hline $\mathrm{F}_{\text {fertilization }}$ & (Tukey' & $77.050)$ & (Tu & $\begin{array}{l}8 * * \\
\text { s test }= \\
20)\end{array}$ & (Tukey & $=0.325)$ & $\begin{array}{r}5 \\
\text { (Tukey' }\end{array}$ & $\begin{array}{l}* * \\
=5.031)\end{array}$ \\
\hline$F_{\text {tillage } X \text { fertilization }}$ & & & & Ans & & & & \\
\hline
\end{tabular}

F-test ratios are from ANOVA. Significant at $* * *$ and $* * *$ indicate significance at $\mathrm{p}=0.05,0.01$ and 0.001 , respectively and ns: not significant. The Tukey's test $(\mathrm{p}=0.05)$ values for tillage system and fertilization are also presented. 
According to the combined analysis, the influence of different tillage systems on total plant nitrogen uptake was found not to be statistically significant, with the values ranging from 331.59 to $365.71 \mathrm{~kg} \mathrm{~N} \mathrm{ha}^{-1}$. Fertilization effect was only significant on this trait. In particular, the highest total nitrogen uptake was achieved in $\mathrm{N} 2$ treatment (437.20-454.93 $\mathrm{kg} \mathrm{N} \mathrm{ha}^{-1}$ ) followed by $\mathrm{N} 1$ treatment (344.59-362.00 $\mathrm{kg} \mathrm{N}^{-1}$ ) and manure (295.40-369.23 $\mathrm{kg} \mathrm{N}$ ha $\left.^{-1}\right)$.

Nitrogen Harvest Index (NHI) did not differ among tillage systems in 2012 and 2013, but significant differences were found during the first year (2011), where the highest value (0.18) was obtained under conventional tillage. Regarding the influence of fertilization, the NHI showed a statistically negative reaction under nitrogen fertilization during the first two years (2011 and 2012) of the experiment. The 3-year average values of NHI were $0.183,0.171,0.159$ and 0.147 for control, manure, N1 and N2 treatment, respectively. NHI had a negative and significant correlation with total plant nitrogen uptake $(r=-0.7322, p<0.001)$.

As shown in Table 3 and according to the combined ANOVA, the tillage system did not affect the apparent nitrogen recovery efficiency (ANRE). Despite the absence of significant effects of tillage systems on ANRE and with the exception of the third year (2013), the highest values (62.28 and 90.39\% in 2011 and 2012, respectively) were found in plots subjected to the conventional system, while, the lowest (54.99 and $79.73 \%$ in 2011 and 2012, respectively) were found under minimum tillage. On the contrary, ANRE was actually influenced by different fertilization treatments with the highest values presented under inorganic fertilization ranging from $79.77 \%$ (N1 treatment in 2011) to $115.48 \%$ (N1 treatment in 2012).
The analysis of variance revealed that nitrogen utilization efficiency NUtE was only affected by fertilization. The differences among all fertilization treatments except for manure and N1 treatment were statistically significant. Results showed that with the increase of nitrogen application, NUtE decreased. During the experimental periods, the highest value (11.08 in 2012) was observed in unfertilized plants, while, the lowest (6.31 in 2012) was found in plants treated with the highest rate $\left(200 \mathrm{~kg} \mathrm{~N} \mathrm{ha}^{-1}\right)$ of nitrogen fertilizer (Table 3).

Nitrogen agronomic efficiency (NAE) was not affected by tillage system but it was significantly influenced by fertilization. In the first two years (2011 and 2012), there were no significant differences between different fertilization regimes, but during the third year of the experiment, fertilization had a great impact on NAE with the mean values being 2.25, 2.92 and 0.57 for application of N2, N1 and manure, respectively. Concerning the average rate of change of nitrogen content in plant (Table 4), fertilization has only a moderately significant effect on this trait. Over the years, there was no increase or decrease in the average rate of each fertilization treatment. The higher range was observed under $\mathrm{N} 2$ treatment with values ranged from 0.025 to $0.027 \mathrm{~N} \%$ day $^{-1}$, followed by $\mathrm{N} 1$ treatment where the values ranged from 0.019 to $0.021 \mathrm{~N} \%$ day $^{-1}$.

The average rate of change of nitrate content in soil was not affected by the tillage system, but it was only influenced by fertilization (Table 5). During the 3-year experiment, the average daily nitrate reduction rates were $-0.0250,-0.0367,-0.0607$ and $-0.1390 \mathrm{ppm} \mathrm{NO}_{3}$ - day ${ }^{-1}$ for control, manure, $\mathrm{N} 1$ and N2 treatment, respectively (Table 5). 
Table 3. Effects of tillage systems (conventional and minimum tillage: CT and MT, respectively) and fertilization (control, inorganic fertilization $100 \mathrm{~kg} \mathrm{~N} \mathrm{ha}^{-1}(\mathrm{~N} 1)$, inorganic fertilization $200 \mathrm{~kg} \mathrm{~N}^{-1}$ (N2) and sheep manure) on nitrogen harvest index (NHI), apparent nitrogen recovery

\begin{tabular}{|c|c|c|c|c|c|c|c|c|}
\hline \multirow[b]{2}{*}{ Fertilization } & \multicolumn{8}{|c|}{ Tillage System } \\
\hline & $\mathrm{CT}$ & MT & $\mathrm{CT}$ & MT & CT & MT & CT & MT \\
\hline 2011 & \multicolumn{2}{|c|}{$\begin{array}{l}\text { Nitrogen Harvest } \\
\text { Index (NHI) }\end{array}$} & \multicolumn{2}{|c|}{$\begin{array}{l}\text { Apparent Nitrogen } \\
\text { Recovery Efficiency } \\
\text { (ANRE) }(\%)\end{array}$} & \multicolumn{2}{|c|}{$\begin{array}{l}\text { Nitrogen Utilization } \\
\text { Efficiency (NUtE) }\end{array}$} & \multicolumn{2}{|c|}{$\begin{array}{c}\text { Nitrogen Agronomic } \\
\text { Efficiency (NAE) }\end{array}$} \\
\hline Control & 0.19 & 0.17 & - & - & 11.70 & 9.96 & - & - \\
\hline N1 & 0.17 & 0.15 & 83.98 & 75.58 & 8.78 & 7.83 & 0.95 & 1.00 \\
\hline $\mathrm{N} 2$ & 0.15 & 0.14 & 91.15 & 83.96 & 6.73 & 6.11 & 0.55 & 0.45 \\
\hline Manure & 0.21 & 0.18 & 11.73 & 5.44 & 11.31 & 10.21 & 0.71 & 0.51 \\
\hline $\mathrm{F}_{\text {tillage }}$ & \multicolumn{2}{|c|}{$\begin{array}{c}5.83^{*} \\
\text { (Tukey's test }=0.027 \text { ) }\end{array}$} & \multicolumn{2}{|c|}{$0.99 \mathrm{~ns}$} & \multicolumn{2}{|c|}{$3.62 \mathrm{~ns}$} & \multicolumn{2}{|c|}{$0.11 \mathrm{~ns}$} \\
\hline $\mathrm{F}_{\text {fertilization }}$ & \multicolumn{2}{|c|}{$\begin{array}{c}8.89 * * \\
(\text { Tukey's test }=0.036)\end{array}$} & \multicolumn{2}{|c|}{$\begin{array}{c}70.36 * * * \\
\text { (Tukey's test }=0.203 \text { ) }\end{array}$} & \multicolumn{2}{|c|}{$\begin{array}{c}13.43 * * \\
\text { (Tukey's test }=2.446)\end{array}$} & \multicolumn{2}{|c|}{$1.32 \mathrm{~ns}$} \\
\hline$F_{\text {tillage }} X$ fertilization & \multicolumn{2}{|c|}{$0.11 \mathrm{~ns}$} & \multicolumn{2}{|c|}{$0.12 \mathrm{~ns}$} & \multicolumn{2}{|c|}{$0.16 \mathrm{~ns}$} & \multicolumn{2}{|c|}{$0.08 \mathrm{~ns}$} \\
\hline 2012 & \multicolumn{2}{|c|}{$\begin{array}{l}\text { Nitrogen Harvest } \\
\text { Index (NHI) }\end{array}$} & \multicolumn{2}{|c|}{$\begin{array}{l}\text { Apparent Nitrogen } \\
\text { Recovery Efficiency } \\
\text { (ANRE) }(\%)\end{array}$} & \multicolumn{2}{|c|}{$\begin{array}{l}\text { Nitrogen Utilization } \\
\text { Efficiency (NUtE) }\end{array}$} & \multicolumn{2}{|c|}{$\begin{array}{l}\text { Nitrogen Agronomic } \\
\text { Efficiency (NAE) }\end{array}$} \\
\hline Control & 0.19 & 0.18 & - & - & 11.69 & 10.48 & - & - \\
\hline N1 & 0.15 & 0.17 & 128.26 & 102.69 & 7.69 & 8.01 & 1.10 & 2.27 \\
\hline $\mathrm{N} 2$ & 0.15 & 0.15 & 108.60 & 99.80 & 6.53 & 6.09 & 1.20 & 1.08 \\
\hline Manure & 0.16 & 0.16 & 34.31 & 36.70 & 8.51 & 7.99 & 0.78 & 1.20 \\
\hline $\mathrm{F}_{\text {tillage }}$ & \multicolumn{2}{|c|}{$0.01 \mathrm{~ns}$} & \multicolumn{2}{|c|}{$1.48 \mathrm{~ns}$} & \multicolumn{2}{|c|}{$2.44 \mathrm{~ns}$} & \multicolumn{2}{|c|}{$1.42 \mathrm{~ns}$} \\
\hline $\mathrm{F}_{\text {fertilization }}$ & \multicolumn{2}{|c|}{$5.01 *$} & \multicolumn{2}{|c|}{$\begin{array}{c}70.98^{* * *} \\
(\text { Tukey's test }=0.279)\end{array}$} & \multicolumn{2}{|c|}{$45.37 * * *$} & \multicolumn{2}{|c|}{$1.06 \mathrm{~ns}$} \\
\hline$F_{\text {tillage } X \text { fertilization }}$ & & & \multicolumn{2}{|c|}{$0.93 \mathrm{~ns}$} & & & & \\
\hline 2013 & $\begin{array}{r}\text { Nitr } \\
\text { In }\end{array}$ & $\begin{array}{l}\text { Harvest } \\
\text { NHI) }\end{array}$ & $\begin{array}{r}\text { Appar } \\
\text { Recove } \\
\text { (AI }\end{array}$ & $\begin{array}{l}\text { itrogen } \\
\text { ficiency } \\
(\%)\end{array}$ & $\begin{array}{l}\text { Nitrog } \\
\text { Effic }\end{array}$ & $\begin{array}{l}\text { lization } \\
\text { NUtE) }\end{array}$ & $\begin{array}{r}\text { Nitrog } \\
\text { Effic }\end{array}$ & $\begin{array}{l}\text { onomic } \\
\text { NAE) }\end{array}$ \\
\hline Control & 0.18 & 0.17 & - & - & 10.76 & 10.01 & - & - \\
\hline N1 & 0.16 & 0.16 & 104.16 & 117.71 & 7.70 & 7.80 & 2.25 & 3.60 \\
\hline $\mathrm{N} 2$ & 0.15 & 0.15 & 98.23 & 105.32 & 6.50 & 6.32 & 2.10 & 2.40 \\
\hline Manure & 0.17 & 0.16 & 25.17 & 30.89 & 7.76 & 7.68 & 0.31 & 0.82 \\
\hline $\mathrm{F}_{\text {tillage }}$ & & & & & & & & \\
\hline$F_{\text {fertilization }}$ & & & $\begin{array}{r}65 \\
\text { (Tukey' }\end{array}$ & $\begin{array}{l}* * \\
=0.252)\end{array}$ & (Tukey & $=2.737)$ & (Tukey & $=1.685)$ \\
\hline$F_{\text {tillage } X \text { fertilization }}$ & & & & & & & & \\
\hline
\end{tabular}

F-test ratios are from ANOVA. Significant at $* * *$ and $* * *$ indicate significance at $p=0.05,0.01$ and 0.001 , respectively and ns: not significant. The Tukey's test $(p=0.05)$ values for tillage system and fertilization are also presented. 
Table 4. Effects of tillage systems (conventional and minimum tillage: CT and MT, respectively) and fertilization (control, inorganic fertilization $100 \mathrm{~kg} \mathrm{~N} \mathrm{ha}^{-1}(\mathrm{~N} 1)$, inorganic fertilization $200 \mathrm{~kg} \mathrm{~N} \mathrm{ha}^{-1}(\mathrm{~N} 2)$ and sheep manure) on rate of change of nitrogen content in quinoa plant $\left(\mathrm{N} \%\right.$ day $\left.^{-1}\right)$.

\begin{tabular}{|c|c|c|c|c|c|c|c|c|c|c|c|c|}
\hline \multirow[b]{2}{*}{ Fertilization } & \multicolumn{12}{|c|}{ Tillage System } \\
\hline & $\mathrm{CT}$ & MT & $\mathrm{CT}$ & MT & $\mathrm{CT}$ & MT & CT & MT & $\mathrm{CT}$ & MT & $\mathrm{CT}$ & MT \\
\hline 2011 & \multicolumn{12}{|c|}{ Rate of Change of Nitrogen Content in Plant $\left(\mathrm{N} \%\right.$ day $\left.^{-1}\right)$} \\
\hline & \multicolumn{2}{|c|}{ 50-25 DAS } & \multicolumn{2}{|c|}{$75-50$ DAS } & \multicolumn{2}{|c|}{ 100-75 DAS } & \multicolumn{2}{|c|}{ 125-100 DAS } & \multicolumn{2}{|c|}{ 150-125 DAS } & \multicolumn{2}{|c|}{ 150-25 DAS } \\
\hline Control & 0.012 & 0.015 & 0.026 & 0.039 & 0.013 & 0.018 & 0.014 & 0.007 & 0.001 & 0.000 & 0.013 & 0.016 \\
\hline N1 & 0.022 & 0.028 & 0.030 & 0.038 & 0.026 & 0.024 & 0.011 & 0.013 & 0.002 & -0.002 & 0.018 & 0.020 \\
\hline N2 & 0.041 & 0.056 & 0.040 & 0.031 & 0.022 & 0.029 & 0.011 & 0.014 & 0.007 & 0.001 & 0.024 & 0.026 \\
\hline Manure & 0.019 & 0.016 & 0.029 & 0.041 & 0.019 & 0.013 & 0.009 & 0.010 & -0.002 & 0.001 & 0.015 & 0.016 \\
\hline $\mathrm{F}_{\text {tillage }}$ & \multicolumn{2}{|c|}{$1.89 \mathrm{~ns}$} & \multicolumn{2}{|c|}{$1.63 \mathrm{~ns}$} & \multicolumn{2}{|c|}{$0.21 \mathrm{~ns}$} & \multicolumn{2}{|c|}{$0.001 \mathrm{~ns}$} & \multicolumn{2}{|c|}{$2.92 \mathrm{~ns}$} & \multicolumn{2}{|c|}{$2.36 \mathrm{~ns}$} \\
\hline $\mathrm{F}_{\text {fertilization }}$ & \multicolumn{2}{|c|}{$\begin{array}{c}15.99 * * \\
\text { (Tukey's test }= \\
0.0169)\end{array}$} & \multicolumn{2}{|c|}{$0.08 \mathrm{~ns}$} & \multicolumn{2}{|c|}{$\begin{array}{c}5.63 * \\
\text { (Tukey's test }= \\
0.0106)\end{array}$} & \multicolumn{2}{|c|}{$1.03 \mathrm{~ns}$} & \multicolumn{2}{|c|}{$\begin{array}{c}4.82 * \\
(\text { Tukey's test }= \\
0.0043)\end{array}$} & \multicolumn{2}{|c|}{$\begin{array}{c}12.33 * * \\
(\text { Tukey's test }= \\
0.0055)\end{array}$} \\
\hline $\begin{array}{l}F_{\text {tillage } X} \\
\text { fertilization }\end{array}$ & \multicolumn{2}{|c|}{$0.99 \mathrm{~ns}$} & \multicolumn{2}{|c|}{$1.23 \mathrm{~ns}$} & \multicolumn{2}{|c|}{$1.68 \mathrm{~ns}$} & \multicolumn{2}{|c|}{$2.10 \mathrm{~ns}$} & \multicolumn{2}{|c|}{$\begin{array}{c}4.68 * \\
(\text { Tukey's test }= \\
0.0076)\end{array}$} & \multicolumn{2}{|c|}{$0.03 \mathrm{~ns}$} \\
\hline \multirow[t]{2}{*}{2012} & & & & Rate & f Chang & of Nitr & gen $\mathrm{Co}$ & ent in $\mathrm{F}$ & nt $(\mathrm{N} \%$ & & & \\
\hline & $50-25$ & DAS & $75-5$ & DAS & $100-7$ & DAS & $125-1$ & DAS & $150-$ & DAS & 150 & DAS \\
\hline Control & 0.017 & 0.014 & 0.023 & 0.032 & 0.017 & 0.015 & 0.012 & 0.005 & 0.001 & 0.003 & 0.014 & 0.014 \\
\hline $\mathrm{N} 1$ & 0.031 & 0.035 & 0.025 & 0.034 & 0.027 & 0.018 & 0.027 & 0.012 & 0.002 & 0.002 & 0.022 & 0.020 \\
\hline $\mathrm{N} 2$ & 0.042 & 0.044 & 0.025 & 0.030 & 0.036 & 0.035 & 0.027 & 0.025 & 0.003 & 0.001 & 0.026 & 0.027 \\
\hline Manure & 0.025 & 0.019 & 0.027 & 0.043 & 0.021 & 0.018 & 0.026 & 0.024 & 0.006 & 0.004 & 0.020 & 0.022 \\
\hline $\mathrm{F}_{\text {tillage }}$ & 0.1 & & & & & & & & & & & \\
\hline $\mathrm{F}_{\text {fertilization }}$ & $\begin{array}{r}18.8 \\
\text { (Tukey } \\
0.0\end{array}$ & $\begin{array}{l}* * * \\
\text { test }= \\
08)\end{array}$ & & & & & & & & & $\begin{array}{r}57 \\
\text { (Tuke } \\
0 .\end{array}$ & $\begin{array}{l}* * * \\
\text { test }= \\
\text { 28) }\end{array}$ \\
\hline $\begin{array}{l}F_{\text {tillage } X} \\
\text { fertilization }\end{array}$ & 0.6 & & & & & & & & & & & \\
\hline 2013 & & & & Rate & Cnang & OI Nitu & gen $\mathrm{Co}$ & ent in $\mathrm{F}$ & nt $(\mathrm{N} \%$ & & & \\
\hline & $50-25$ & DAS & $75-5$ & DAS & $100-7$ & DAS & $125-1$ & DAS & $150-$ & DAS & $150-$ & DAS \\
\hline Control & 0.014 & 0.020 & 0.029 & 0.027 & 0.013 & 0.025 & 0.016 & 0.005 & 0.003 & 0.002 & 0.015 & 0.016 \\
\hline N1 & 0.019 & 0.022 & 0.029 & 0.033 & 0.033 & 0.030 & 0.017 & 0.029 & 0.002 & -0.001 & 0.020 & 0.022 \\
\hline $\mathrm{N} 2$ & 0.017 & 0.031 & 0.039 & 0.033 & 0.032 & 0.030 & 0.034 & 0.035 & 0.002 & 0.001 & 0.025 & 0.026 \\
\hline Manure & 0.014 & 0.019 & 0.032 & 0.030 & 0.017 & 0.028 & 0.028 & 0.020 & 0.003 & 0.002 & 0.019 & 0.020 \\
\hline$F_{\text {tillage }}$ & 4.5 & & & & 1.1 & & & & & & & \\
\hline $\mathrm{F}_{\text {fertilization }}$ & & & & & & & & & & & & \\
\hline $\begin{array}{l}F_{\text {tillage } X} \\
\text { fertilization }\end{array}$ & 0.4 & & & & & & & & & & $\begin{array}{r}0 \\
\text { (Tuke } \\
0 .\end{array}$ & $\begin{array}{l}\text { ns } \\
\text { test }= \\
\text { 17) }\end{array}$ \\
\hline
\end{tabular}

F-test ratios are from ANOVA. Significant at $* * *$ and $* * *$ indicate significance at $p=0.05,0.01$ and 0.001 , respectively and ns: not significant. The Tukey's test $(p=0.05)$ values for tillage system and fertilization are also presented. 
Table 5. Effects of tillage systems (conventional and minimum tillage: CT and MT, respectively) and fertilization (control, inorganic fertilization $100 \mathrm{~kg} \mathrm{~N} \mathrm{ha}^{-1}(\mathrm{~N} 1)$, inorganic fertilization $200 \mathrm{~kg} \mathrm{~N}^{-1}$ (N2) and sheep manure) on rate of change of nitrates in soil $\left(\mathrm{ppm} \mathrm{NO}_{3}-\right.$ day $\left.^{-1}\right)$.

\begin{tabular}{|c|c|c|c|c|c|c|c|c|c|c|c|c|}
\hline \multirow[b]{2}{*}{ Fertilization } & \multicolumn{12}{|c|}{ Tillage System } \\
\hline & CT & MT & CT & MT & CT & MT & CT & MT & $\mathrm{CT}$ & MT & CT & MT \\
\hline \multirow[t]{2}{*}{2011} & \multicolumn{12}{|c|}{ Rate of Change of Nitrates in Soil $\left(\mathrm{ppm} \mathrm{NO}_{3}^{-}\right.$day $\left.^{-1}\right)$} \\
\hline & \multicolumn{2}{|c|}{$50-25$ DAS } & \multicolumn{2}{|c|}{$75-50$ DAS } & \multicolumn{2}{|c|}{ 100-75 DAS } & \multicolumn{2}{|c|}{ 125-100 DAS } & \multicolumn{2}{|c|}{ 150-125 DAS } & \multicolumn{2}{|c|}{ 150-25 DAS } \\
\hline Control & 0.000 & -0.040 & 0.000 & 0.020 & 0.020 & -0.020 & -0.080 & 0.000 & 0.000 & 0.040 & -0.012 & 0.000 \\
\hline N1 & -0.060 & -0.080 & 0.000 & 0.020 & -0.100 & 0.040 & -0.060 & -0.040 & 0.000 & 0.000 & -0.044 & -0.012 \\
\hline $\mathrm{N} 2$ & -0.060 & 0.020 & -0.120 & -0.180 & -0.020 & -0.180 & -0.200 & -0.220 & -0.040 & -0.040 & -0.124 & -0.120 \\
\hline Manure & -0.020 & -0.040 & -0.100 & 0.000 & -0.060 & -0.040 & 0.020 & -0.020 & -0.020 & -0.020 & -0.036 & -0.024 \\
\hline $\mathrm{F}_{\text {tillage }}$ & \multicolumn{2}{|c|}{$0.01 \mathrm{~ns}$} & \multicolumn{2}{|c|}{$0.99 \mathrm{~ns}$} & \multicolumn{2}{|l|}{$0.60 \mathrm{~ns}$} & \multicolumn{2}{|l|}{$0.17 \mathrm{~ns}$} & \multicolumn{2}{|c|}{$0.29 \mathrm{~ns}$} & \multicolumn{2}{|c|}{$2.92 \mathrm{~ns}$} \\
\hline$F_{\text {fertilization }}$ & \multicolumn{2}{|c|}{$0.63 \mathrm{~ns}$} & \multicolumn{2}{|c|}{$\begin{array}{c}14.25^{* *} \\
(\text { Tukey's test }= \\
0.0999)\end{array}$} & $3.50 \mathrm{~ns}$ & \multicolumn{3}{|c|}{$\begin{array}{c}14.28 * * \\
(\text { Tukey's test }=0.1014)\end{array}$} & \multicolumn{2}{|c|}{$1.90 \mathrm{~ns}$} & \multicolumn{2}{|c|}{$\begin{array}{c}34.44 * * * \\
\text { (Tukey's test }= \\
0.0373)\end{array}$} \\
\hline $\begin{array}{l}F_{\text {tillage } X} \\
\text { fertilization }\end{array}$ & \multicolumn{2}{|c|}{$0.81 \mathrm{~ns}$} & \multicolumn{2}{|c|}{$1.23 \mathrm{~ns}$} & $0.70 \mathrm{~ns}$ & \multicolumn{3}{|c|}{$1.17 \mathrm{~ns}$} & \multicolumn{2}{|c|}{$0.29 \mathrm{~ns}$} & \multicolumn{2}{|c|}{$0.46 \mathrm{~ns}$} \\
\hline \multirow[t]{2}{*}{2012} & & & & & of Chan & f Nitrate & in Soil (p & $\mathrm{n} \mathrm{NO}_{3}^{-}$ & $\left.\mathrm{y}^{-1}\right)$ & & & \\
\hline & $50-$ & DAS & $75-5$ & DAS & 100 & DAS & $125-10$ & DAS & 150-1 & DAS & 150 & DAS \\
\hline Control & 0.080 & 0.000 & -0.060 & 0.060 & 0.060 & 0.060 & -0.180 & -0.120 & -0.020 & -0.100 & -0.024 & -0.020 \\
\hline N1 & 0.200 & 0.000 & -0.180 & -0.020 & -0.060 & 0.140 & -0.260 & -0.140 & -0.040 & -0.240 & -0.068 & -0.052 \\
\hline $\mathrm{N} 2$ & 0.120 & 0.160 & -0.140 & -0.080 & -0.220 & -0.100 & -0.280 & -0.360 & -0.080 & -0.100 & -0.120 & -0.096 \\
\hline Manure & 0.080 & 0.040 & -0.020 & 0.080 & -0.060 & 0.000 & -0.120 & -0.200 & -0.080 & -0.040 & -0.040 & -0.024 \\
\hline $\mathrm{F}_{\text {tillage }}$ & & & $\begin{array}{r}10 \\
\text { Tuke } \\
0 .\end{array}$ & $\begin{array}{l}2 * \\
\text { test }= \\
4)\end{array}$ & & & & & & & & \\
\hline$F_{\text {fertilization }}$ & & & $\begin{array}{r}4 \\
\text { (Tuke } \\
0 .\end{array}$ & test $=$ & & & & & & & $\begin{array}{r}8 \\
\text { (Tuke } \\
0 .\end{array}$ & $\begin{array}{l}* * \\
\text { test }= \\
0)\end{array}$ \\
\hline $\begin{array}{l}F_{\text {tillage } X} \\
\text { fertilization }\end{array}$ & & & $0.38 \mathrm{~ns}$ & & & & & & & & & \\
\hline 2013 & & & & & of Chan & f Nitrate & in Soil (p & $\mathrm{n} \mathrm{NO}_{3}^{-}$ & & & & \\
\hline & $50-$ & DAS & $75-5$ & DAS & 100 & DAS & $125-1$ & DAS & $150-1$ & DAS & 150 & DAS \\
\hline Control & 0.040 & 0.000 & -0.020 & 0.040 & 0.000 & -0.100 & -0.160 & -0.080 & -0.100 & -0.080 & -0.048 & -0.044 \\
\hline N1 & 0.040 & 0.040 & -0.080 & -0.060 & -0.140 & -0.080 & -0.200 & -0.280 & -0.080 & -0.100 & -0.092 & -0.096 \\
\hline $\mathrm{N} 2$ & -0.060 & -0.140 & -0.140 & -0.120 & -0.280 & -0.240 & -0.220 & -0.400 & -0.180 & -0.100 & -0.176 & -0.200 \\
\hline Manure & 0.120 & 0.100 & 0.020 & -0.040 & 0.000 & -0.100 & -0.200 & -0.200 & -0.100 & -0.080 & -0.032 & -0.064 \\
\hline $\mathrm{F}_{\text {tillage }}$ & & & & & & & & & & & & \\
\hline$F_{\text {fertilization }}$ & & & & & (Tukey's & $=0.1631)$ & & & & & $\begin{array}{r}13 \\
\text { (Tuke } \\
0 .\end{array}$ & $\begin{array}{l}* * \\
\text { test }= \\
9)\end{array}$ \\
\hline $\begin{array}{l}F_{\text {tillage } X} \\
\text { fertilization }\end{array}$ & & & & & & & & & & & & \\
\hline
\end{tabular}

F-test ratios are from ANOVA. Significant at * $* *$ and $* * *$ indicate significance at $p=0.05,0.01$ and 0.001 , respectively and ns: not significant. The Tukey's test $(p=0.05)$ values for tillage system and fertilization are also presented. 
Table 6. Combined analysis of variance (F values) for all studied characteristics of nitrogen uptake and utilization efficiency of quinoa crop.

\begin{tabular}{|c|c|c|c|c|c|c|c|}
\hline $\begin{array}{l}\text { Source of } \\
\text { Variance }\end{array}$ & df & $\begin{array}{c}\text { Biomass N } \\
\text { Content }\end{array}$ & $\begin{array}{c}\text { Biomass N } \\
\text { Yield }\end{array}$ & $\begin{array}{l}\text { Total Plant N } \\
\text { Uptake }\end{array}$ & $\begin{array}{l}\text { Seed N } \\
\text { Content }\end{array}$ & \multicolumn{2}{|c|}{$\begin{array}{l}\text { Seed N } \\
\text { Yield }\end{array}$} \\
\hline Year & 2 & $91.55 * * *$ & $1.36 \mathrm{~ns}$ & $1.63 \mathrm{~ns}$ & $4.73 *$ & \multicolumn{2}{|c|}{$1.89 \mathrm{~ns}$} \\
\hline Tillage & 1 & $0.68 \mathrm{~ns}$ & $0.02 \mathrm{~ns}$ & $0.01 \mathrm{~ns}$ & $0.05 \mathrm{~ns}$ & \multicolumn{2}{|c|}{$2.92 \mathrm{~ns}$} \\
\hline Fertilization & 3 & $18.46^{* * *}$ & $46.19 * * *$ & $51.49 * * *$ & $35.91 * * *$ & \multicolumn{2}{|c|}{$23.43 * * *$} \\
\hline Year x Tillage & 2 & $0.67 \mathrm{~ns}$ & $0.48 \mathrm{~ns}$ & $0.39 \mathrm{~ns}$ & $2.31 \mathrm{~ns}$ & \multicolumn{2}{|c|}{$0.82 \mathrm{~ns}$} \\
\hline $\begin{array}{c}\text { Year } \mathrm{x} \\
\text { Fertilization }\end{array}$ & 6 & $17.19^{* * *}$ & $1.09 \mathrm{~ns}$ & $1.06 \mathrm{~ns}$ & $0.96 \mathrm{~ns}$ & \multicolumn{2}{|c|}{$0.66 \mathrm{~ns}$} \\
\hline $\begin{array}{c}\text { Tillage } \mathrm{x} \\
\text { Fertilization }\end{array}$ & 3 & $1.18 \mathrm{~ns}$ & $0.06 \mathrm{~ns}$ & $0.04 \mathrm{~ns}$ & $0.63 \mathrm{~ns}$ & \multicolumn{2}{|c|}{$0.13 \mathrm{~ns}$} \\
\hline $\begin{array}{l}\text { Year x Tillage } \\
\mathrm{x} \text { Fertilization }\end{array}$ & 6 & $0.37 \mathrm{~ns}$ & $0.10 \mathrm{~ns}$ & $0.11 \mathrm{~ns}$ & $0.05 \mathrm{~ns}$ & \multicolumn{2}{|c|}{$0.10 \mathrm{~ns}$} \\
\hline $\begin{array}{l}\text { Source of } \\
\text { Variance }\end{array}$ & df & $\begin{array}{l}\text { N Harvest } \\
\text { Index } \\
\text { (NHI) }\end{array}$ & $\begin{array}{l}\text { Apparent N } \\
\text { Recovery } \\
\text { Efficiency } \\
\text { (ANRE) }\end{array}$ & $\begin{array}{c}\text { N Utilization } \\
\text { Efficiency } \\
\text { (NUtE) }\end{array}$ & $\begin{array}{c}\mathrm{N} \\
\text { Agronomic } \\
\text { Efficiency } \\
\text { (NAE) }\end{array}$ & $\begin{array}{l}\text { Rate of } \\
\text { Change } \\
\text { of N } \\
\text { Content } \\
\text { in Plant }\end{array}$ & $\begin{array}{c}\text { Rate of } \\
\text { Change of } \\
\text { Nitrates in } \\
\text { Soil }\end{array}$ \\
\hline Year & 2 & $0.44 \mathrm{~ns}$ & $11.23 * * *$ & $3.10 \mathrm{~ns}$ & $9.45 * * *$ & $3.63^{*}$ & $13.22 * * *$ \\
\hline Tillage & 1 & $1.85 \mathrm{~ns}$ & $0.397 \mathrm{~ns}$ & $3.09 \mathrm{~ns}$ & $2.67 \mathrm{~ns}$ & $2.19 \mathrm{~ns}$ & $0.44 \mathrm{~ns}$ \\
\hline Fertilization & 3 & $5.92 * *$ & $202.52 * * *$ & $29.04 * * *$ & $21.45 * * *$ & $\begin{array}{c}48.23^{* *} \\
*\end{array}$ & $41.38 * * *$ \\
\hline Year x Tillage & 2 & $0.79 \mathrm{~ns}$ & $1.53 \mathrm{~ns}$ & $0.59 \mathrm{~ns}$ & $1.09 \mathrm{~ns}$ & $1.35 \mathrm{~ns}$ & $1.46 \mathrm{~ns}$ \\
\hline Year x Tillage & 2 & $0.79 \mathrm{~ns}$ & $1.53 \mathrm{~ns}$ & $0.59 \mathrm{~ns}$ & $1.09 \mathrm{~ns}$ & $1.35 \mathrm{~ns}$ & $1.46 \mathrm{~ns}$ \\
\hline Year $\mathrm{x}$ Tillage & 2 & $0.79 \mathrm{~ns}$ & $1.53 \mathrm{~ns}$ & $0.59 \mathrm{~ns}$ & $1.09 \mathrm{~ns}$ & $1.35 \mathrm{~ns}$ & $1.46 \mathrm{~ns}$ \\
\hline $\begin{array}{c}\text { Year } \mathrm{x} \\
\text { Fertilization }\end{array}$ & 6 & $0.94 \mathrm{~ns}$ & $1.73 \mathrm{~ns}$ & $1.70 \mathrm{~ns}$ & $3.67 \mathrm{~ns}$ & $1.43 \mathrm{~ns}$ & $1.51 \mathrm{~ns}$ \\
\hline $\begin{array}{c}\text { Tillage } \mathrm{x} \\
\text { Fertilization }\end{array}$ & 3 & $0.25 \mathrm{~ns}$ & $0.22 \mathrm{~ns}$ & $0.44 \mathrm{~ns}$ & $1.34 \mathrm{~ns}$ & $0.01 \mathrm{~ns}$ & $0.19 \mathrm{~ns}$ \\
\hline $\begin{array}{l}\text { Year x Tillage } \\
\text { x Fertilization }\end{array}$ & 6 & $0.04 \mathrm{~ns}$ & $0.54 \mathrm{~ns}$ & $0.05 \mathrm{~ns}$ & $0.30 \mathrm{~ns}$ & $0.21 \mathrm{~ns}$ & $0.22 \mathrm{~ns}$ \\
\hline
\end{tabular}

F-test ratios are from ANOVA. Significant at *,** and *** indicate significance at $p=0.05,0.01$ and 0.001 , respectively and ns: not significant.

\section{Discussion}

The results of the present study indicated that the biomass nitrogen accumulation was linearly and positive affected by nitrogen supply. The maximum biomass nitrogen concentration was on average $4.14 \%$ and observed under the highest nitrogen rate (200 $\left.\mathrm{kg} \mathrm{N} \mathrm{ha}^{-1}\right)$. Similarly, Elbehri et al. (1993) reported that in amaranth, nitrogen concentration of biomass was also linearly increased along with the rates of nitrogen fertilization.

The biomass nitrogen yield was slightly higher in plants grown in soils subjected under conventional than minimum tillage, however, this difference was not statistically significant. These results are in agreement with those obtained by Małecka and Blecharczyk (2008) who reported that in spring barley conventional tillage achieved slightly but significantly higher biomass nitrogen accumulation than reduced tillage. On the other hand, Pushpa Devi et al. (2014) found that nitrogen concentration in straw of wheat were higher by 9 to $30 \%$ in reduced tillage as compared to conventional tillage. A very important nitrogen yield enhancement was observed in plants treated with the highest rate of nitrogen fertilizer (N2). The large difference between the values of N2 treatment and control $\left(161 \mathrm{~kg} \mathrm{~N} \mathrm{ha}^{-1}, 184 \mathrm{~kg} \mathrm{~N} \mathrm{ha}^{-1}\right.$ and $178 \mathrm{~kg} \mathrm{~N} \mathrm{ha}^{-1}$ for 2011, 2012 and 2013, respectively), revealed that quinoa responds well to nitrogen fertilization and has the ability to accumulate nitrogen 
in biomass. This finding is in accordance with a previous study by Razzaghi et al. (2012).

Under the experimental conditions, seed nitrogen content and yield showed the same pattern as biomass nitrogen content and yield, indicating that the higher the nitrogen supply, the higher was the nitrogen accumulation in seed. In another study, Thanapornpoonpong (2004) also demonstrated the positive effect of nitrogen fertilization on seed nitrogen content of quinoa crop. Similarly, increase of grain nitrogen accumulation and concentation was caused in other crops (wheat, soybean) by nitrogen fertilization (Hayati et al., 1996; Noulas et al., 2004). Moreover, Delogu et al. (1998) demonstrated that after application of 80 $\mathrm{kg} \mathrm{N} \mathrm{ha}^{-1}$ in barley, the nitrogen content in grain was enhanced by about $18 \%$ compared to the control.

Nitrogen fertilization has also a positive effect on total nitrogen uptake. During these three years, total nitrogen uptake increased linearly with increasing nitrogen fertilizer rate. In a previous study, it was observed that total nitrogen uptake was nearly doubled from $82.6 \mathrm{~kg} \mathrm{~N}^{-1}$ under unfertilized treatment to $161.3 \mathrm{~kg} \mathrm{~N} \mathrm{ha}^{-1}$ under fertilization of $120 \mathrm{~kg} \mathrm{~N} \mathrm{ha}^{-1}$ (Schulte auf'm Erley et al., 2005).

Nitrogen Harvest Index (NHI) is an important index for measuring the retranslocation efficiency of absorved nitrogen from vegetative parts of a plant to grain (Fageria and Baligar, 2005). The NHI was 30-60\% higher in control and manure plots than in plots treated with inorganic fertilizers. These values are relatively lower compared with other crops (Kaul et al., 1996) due to the small ratio of seed yield to the total above-ground biomass and seed yield that characterized this crop. Thus, while increasing the seed yield and seed nitrogen yield, the denominator of ratio is considerably higher. Apparent Nitrogen Recovery Efficiency (ANRE) is defined as the difference in nitrogen uptake in above-ground parts of the plant between the fertilized and unfertilized crop relative to the quality of nitrogen applied and is the most preferred NUE index to access the nitrogen response of the crop. Moreover, ANRE varied among the plant growth stages. Specifically, in maize, it was found that ANRE decreased between the start of grain filling stage and physiological maturity due to increase in nitrogen losses on account of leaf death (Shah et al., 2016). In our results, the values observed in inorganic fertilization treatments during 2012 and 2013 were greater than $100 \%$ (ANRE $>100 \%)$, which indicates that crop absorbed not only fertilizer nitrogen but also available soil nitrogen. These results are in contrast with results reported by Thanapornpoonpong (2004). Nitrogen utilization efficiency (NUtE) defines the relationship between crop yield and total nitrogen absorbed by the biomass and grains of the plant. NUtE declined with the increase in nitrogen supply. The values were up to $40 \%$ lower in inorganic treatments than in the control. Schulte auf'm Erley et al. (2005) reported that this indicator was not influenced by the nitrogen rate in a quinoa crop. NUtE had a negative and significant correlation with biomass nitrogen yield ( $r=-0,912, p<0,001)$, demonstrating a lower utilization of nitrogen for grain production and higher for biomass production.

Nitrogen Agronomic Efficiency (NAE) relates the ability of the plant to increase economical (grain) yield with the nitrogen applied (Craswell and Godwin, 1984). The increase in this indicator suggests higher grain yields. In our results, the optimum application rate of fertilizer $\mathrm{N}$ for optimal economic (grain) yield in a quinoa crop is $100 \mathrm{~kg} \mathrm{~N} \mathrm{ha}^{-1}$ (N1). A similar behavior of NAE has been reported by several researchers in other crops (Artacho et al., 2009; Baitilwake et al., 2011).

The average rate of change in nitrogen content in plant describes the average growth rate of nitrogen in the plant and its potential to accumulate nitrogen. The higher values ranged from 0.025 to $0.027 \mathrm{~N} \%$ day 
were achieved in N2 treatment. These values are considered to be higher in comparison with other crops that makes quinoa as a crop with high nitrogen accumulation rate in the aerial biomass (Ye et al., 2007; Geissler et al., 2015). In addition, the average range of change in nitrogen content in the plant had a positive and significant correlation with root density and dry matter of quinoa (Kakabouki, 2016). This is associated with the rapid supply of quinoa with nitrogen through the root system and with the needs of aboveground parts for nitrogen due to the rapid growth rate of the plant (Jacobsen et al., 1994).

Concerning the average rate of change of nitrate content in soil, the lowest value (-0.1390 ppm $\mathrm{NO}_{3}-$ day $^{-1}$ ) was found in N2 treatment, while, the lowest value (-0.025 ppm $\mathrm{NO}_{3}-$ day $\left.^{-1}\right)$ was observed in unfertilized (control) plots. The average daily nitrate reduction rate in soil in $\mathrm{N} 2$ treatment $\left(200 \mathrm{~kg} \mathrm{~N} \mathrm{ha}^{-1}\right)$ was five times greater than in control $\left(0 \mathrm{~kg} \mathrm{~N} \mathrm{ha}^{-1}\right)$, consisting one of the biggest differences (Mackova et al., 2006; Willey, 2007).

The rates of change of nitrate reduction in soil and nitrogen increase in the plant, are associated indicators that describe the soil-plant system as far as nitrogen is concerned. The high rate of average daily nitrogen increase in plants and the high rate of average daily reduction in soils treated with high amounts of inorganic fertilizers, confirm the potential of quinoa and its ability to can be also used as a phytoremediation plant. The close relationship between these two rates is also confirmed by the high linear correlation coefficient $(r=-0,726, p<0,001)$.

\section{Conclusions}

The present results confirmed that nitrogen uptake and use efficiency in quinoa crop was only significantly affected by fertilization. Biomass nitrogen content, biomass nitrogen yield, seed nitrogen con- tent, seed nitrogen yield and total plant nitrogen uptake increased with the increase of nitrogen application rate. Nitrogen harvest index (NHI) and nitrogen utilization efficiency (NUtE) declined at high rates of nitrogen supply. The apparent nitrogen recovery efficiency (ANRE) was actually influenced by fertilization, with the higher values observed in inorganic fertilized treatments. Moreover, rates of nitrogen higher than $100 \mathrm{~kg} \mathrm{~N}^{-1}$ did not increase the nitrogen agronomic efficiency (NAE). The average rate of change in nitrogen content in plant and rate of change of nitrates in soil increased and decreased, respectively, with increasing nitrogen application rate. As a conclusion, quinoa has a high capacity to take up nitrate from the soil, but presents lower nitrogen remobilization from the vegetative parts into the seeds under high nitrogen supply.

\section{References}

Artacho, P., Bonomelli, C., Meza, F. 2009. Nitrogen Application in irrigated rice grown in Mediterranean conditions: Effects on grain yield, dry matter production, nitrogen uptake, and nitrogen use efficiency. J. Plant Nutr. 32: 1574-1593.

Baitilwake, M.A., De Bolle, S., Salomez, J., Mrema, J.P., De Neve, S. 2011. Effects of manure nitrogen on vegetables' yield and nitrogen efficiency in Tanzania. Int. J. Plant Prod. 5, 417-430.

Benlhabib, O., Yazar A., Qadir M., Lourenço E., Jacobsen, S.-E. 2014. How can we improve Mediterranean cropping systems? Journal of Agronomy and Crop Science. 200, 325-332.

Bilalis D., Kakabouki I., Karkanis A., Travlos I., Triantafyllidis V., Hela D. 2012. Seed and saponin production of organic quinoa (Chenopodium quinoa Willd.) for different tillage and fertilization. Not. Bot. Horti Agrob. 40, 42-46. 
Craswell, E.T., Godwin, D.C. 1984. The efficiency of nitrogen fertilizers applied to cereals in different climates. Adv. Plant Nutr. 1, 1-55.

Delogu, G., Cattivelli, L., Pecchioni, N., De Falcis, D., Maggiore, T., Stanca, A.M. 1998. Uptake and agronomic efficiency of nitrogen in winter barley and winter wheat. Eur. J. Agron. 9, 11-20.

Elbehri, A., Putnam, D.H., Schmitt, M. 1993. Nitrogen fertilizer and cultivar effects on yield and nitrogen-use efficiency of grain amaranth. Agron. J. $85,120-128$.

Fageria, N.K., Baligar, V.C. 2005. Enhancing nitrogen use efficiency in crop plants. Adv. Agron. 88, 97- 185 .

Geissler, N., Hussin, S., El-Far, M., Koyro, H. 2015. Elevated atmospheric $\mathrm{CO}_{2}$ concentration leads to different salt resistance mechanisms in a $\mathrm{C} 3$ (Chenopodium quinoa) and a C4 (Atriplex nummularia) halophyte. Envir. \& Exper. Bot. 118, $67-77$.

Hayati, R., Egli, D.B., Crafts-Brandner, S.J. 1996. Independence of nitrogen supply and seed growth in soybean: studies using an in vitro culture system. J. Exper. Botany. 47, 33-40.

Jacobsen, S.-E., Jørgensen, I., Stølen, O. 1994. Cultivation of quinoa (Chenopodium quinoa) under temperate climatic conditions in Denmark, The J. Agric. Sc. 122, 47-52.

Jacobsen, S.E. 2003. The worldwide potential for quinoa (Chenopodium quinoa Willd.). Food Rev. Intern. 19, 167-177.

Kakabouki, I., Bilalis, D., Karkanis, A., Zervas, G., Tsiplakou, E., Hela, D. 2014. Effects of fertilization and tillage system on growth and crude protein content of quinoa (Chenopodium quinoa Willd.): An alternative forage. Em. J. Food and Agric. 26, 18-24.
Kakabouki I. 2016. "Remediation possibility of nitrate-contaminated soils using Quinoa crop" $\mathrm{PhD}$ Thesis. University of Patras, Greece, pgs 178.

Kaul H.P., Aufhammer W., Wagner W. 1996. Dry matter and nitrogen accumulation and residues of oil and protein crops. Eur. J. Agron. 5, 137-147.

Keeney, D.R., Nelson, D.W. 1982. Nitrogen-Inorganic Forms. In: Page A L et al. (Eds), Methods of Soil Analysis. Part 2. Agronomy Monographs 9. ASA and SSSA, Madison, WI, 643-649.

Lobos Ortega, I., Alfaro, M., Martinez-Lagos, J. 2016. Soil nitrogen contribution to grasslands yield in southern Chile its implications for nitrogen use efficiency. J. Soil Sci. Plant Nutr. 16, 310-322.

Koziol, M. 1992. Chemical composition and nutritional evaluation of Quinoa (Chenopodium quinoa Willd.). J.of Food Comp. \& Anal. 5, 35-68.

Mackova M., Dowling D., Macek T. 2006. Phytoremediation - Rhizoremediation. Published by Springer, P.O. Box 17, 3300 AA Dordrecht, The Netherlands.

Mahler, R.L., Koehler, F.E., Lutcher, L.K. 1994. Soils. Nitrogen source, timing of application, and placement: effects on winter wheat production. Agron. J. 86, 637-642.

Małecka, I., Blecharczyk. I. 2008. Effects of tillage systems, mulches and nitrogen fertilization on spring barley (Hordeum vulgare). Agron. Res. 6, 517-529.

Montemurro, F., Diacono, M. 2016. Towards a Better Understanding of Agronomic Efficiency of $\mathrm{Ni}$ trogen: Assessment and Improvement Strategies. Agronomy. 6, 31 .

Noulas, C., P. Stamp, A. Soldati, M. Liedgens. 2004. Nitrogen use efficiency of spring wheat genotypes under field and lysimeter conditions. J. Agron. \& Crop Sc. 190, 111-118. 
Pushpa D., Aggarwal A., Gupta S.R. 2014. Carbon Accumulation, Nitrogen Uptake and Mycorrhizal Root Colonization in a Tropical Rice-Wheat System in Northern India. Ind. J. Sc. 11, 21-31.

Razzaghi, F., Plauborg, F., Jacobsen, S.-E., Jensen, C. R., Andersen, M.N. 2012. Effect of nitrogen and water availability of three soil types on yield, radiation use efficiency and evapotranspiration in fieldgrown quinoa. Agric.Water Manag. 109, 20-29.

Shah, G.M., Shah, G.A., Groot, J.C.J., Raza, M.A.S., Shahid, N., Lantinga, E.A. 2016. Maize nitrogen recovery and dry matter production as affected by application of solid cattle manure subjected to various storage conditions. J. Soil Sci. Plant Nutr. 16, 591-603.

Sinclair, T.R., de Wit, C.T. 1975. Photosynthate and nitrogen requirements for seed production by various crops. Science. 18, 565-567.

Schulte-auf'm-Erley G., Kaul H.P., Kruse M, Aufhammer W. 2005. Yield and nitrogen utilization efficiency of the pseudocereals amaranth, quinoa and buckwheat under differing nitrogen fertilization. Eur. J. Agron. 22, 95-100.

Thanapornpoonpong, S. 2004. Effect of nitrogen fertilizer on nitrogen assimilation and seed quality of amaranth (Amaranthus spp.) and quinoa (Chenopodium quinoa Willd), Doctoral Dissertation, Doctor of Agricultural Sciences of the Faculty of Agricultural Sciences, Institute of Agricultural Chemistry, Georg-August-University of Göttingen.
Thanapornpoonpong S., Vearasilp, S., Pawelzik, E., Gorinstein, S. 2008. Influence of various nitrogen applications on protein and amino acid profiles of amaranth and quinoa. J. Agric. \& Food Chem. 56, 11464-11470.

Vega-Gálvez, A., Miranda M., Vergara J., Uribe E., Puente L., Martinez E.A. 2010. Nutrition facts and functional potential of quinoa (Chenopodium quinoa Willd.). An ancient Andean grain: a review. J. Sc Food \& Agric. 90, 2541-2547.

Willey, N. 2007. Phytoremediation -Methods and Reviews. Humana Press Inc. 999 Riverview Drive, Suite 208 Totowa, New Jersey 07512

Ye, Q., Zhang, H., Wei, H., Zhang, Y., Wang, B., Xia, K., Huo, Z., Dai, Q., Xu, K. 2007. Effects of nitrogen fertilizer on nitrogen use efficiency and yield of rice under different soil conditions. Fr. Agric. Ch. 1, 30-36.

Zemenchik, R.A., Albrecht, K.A. 2002. Nitrogen use efficiency and apparent nitrogen recovery of Kentucky bluegrass, smooth bromegrass, and orchardgrass. Agron. J. 94, 421-428. 